\title{
Fatores associados à notificação de maus-tratos em crianças e adolescentes na atenção básica
}

\author{
Factors associated with the reporting of child \\ and adolescent abuse in primary healthcare
}

Gracyelle Alves Remigio Moreira ${ }^{1}$
Luiza Jane Eyre de Souza Vieira ${ }^{1}$
Suely Ferreira Deslandes ${ }^{2}$
Maria Augediva Jucá Pordeus ${ }^{3}$
Isabelle da Silva Gama ${ }^{4}$
Aline Veras Morais Brilhante ${ }^{1}$
${ }^{1}$ Programa de Pós-

Graduação em Saúde

Coletiva, Universidade de Fortaleza. Av. Washington Soares 1321/Bloco S/Sala 1, Edson Queiroz. 60.811-905 Fortaleza Ceará Brasil. gracyremigio@gmail.com ${ }^{2}$ Instituto Fernandes Figueira, Fundação Oswaldo Cruz.

${ }^{3}$ Centro de Ciências da Saúde, Universidade de Fortaleza.

${ }^{4}$ Departamento de Saúde Comunitária, Faculdade de Medicina, Universidade Federal do Ceará.

\begin{abstract}
This study aimed to analyze factors associated with the reporting of child and adolescent abuse in primary healthcare. It is a cross-sectional study conducted with 381 professionals (physicians, nurses and dental surgeons) from three healthcare regions of the state of Ceara, Brazil. It used a self-administered questionnaire asking for socio-demographic data, vocational education and training, instrumentation and knowledge on the issue, identification and reporting of abuse cases. Bivariate and multivariate analyses using logistic regression were performed. There was a frequency of $17 \%$ of abuse reporting by basic healthcare professionals. The multivariate analysis showed that participating in the training on the issue $(O R=$ 2.18; CI95\% = 1.05-4.53), being familiar with the reporting form $(\mathrm{OR}=3.03$; $\mathrm{CI} 95 \%=1.47-$ $6.24)$ and knowing how to refer the case properly $(\mathrm{OR}=2.55 ; \mathrm{CI} 95 \%=1.01-6.43)$ increased the chance of professionals reporting child and adolescent abuse. The variables identified in the final model showed a direct association between the act of reporting and matters related to knowledge and training on the issue. Investments in the training of healthcare professionals are required in order to favor the qualification of the practice for this public health problem.
\end{abstract}

Key words Reporting, Violence, Child, Adolescent, Primary healthcare
Resumo Objetivou-se analisar os fatores associados à notificação de maus-tratos em crianças e adolescentes na atenção básica. Estudo transversal com 381 profissionais (médicos, enfermeiros e cirurgiões-dentistas), de três Regiões de Saúde do Estado do Ceará, Brasil. Foi utilizado um questionário autoaplicável sobre dados sociodemográficos, formação profissional, instrumentação e conhecimento sobre o tema, identificação e notificação de casos de maus-tratos. Análises bivariada e multivariada por regressão logística foram realizadas. Verificou-se que a frequência da notificação de maus-tratos pelos profissionais da atenção básica foi de 17,0\%. A análise multivariada mostrou que participar de treinamento sobre o tema (OR $=2,18 ;$ IC95\% $=1,05-4,53)$, conhecer a ficha de notificação $(O R=3,03$; IC95\% = 1,47-6,24) e saber encaminhar os casos $(\mathrm{OR}=2,55$; IC95\% = 1,01-6,43) aumentou a chance dos profissionais notificarem situações de maus-tratos em crianças $e$ adolescentes. As variáveis identificadas no modelo final sinalizam associação direta do ato de notificar com questões relativas ao conhecimento e capacitação na temática. Sugerem-se investimentos na formação dos profissionais de saúde com a finalidade de favorecer a qualificação da prática diante desse problema de saúde pública.

Palavras-chave Notificação, Violência, Criança, Adolescente, Atenção básica 


\section{Introdução}

Crianças e adolescentes são suscetíveis e vulneráveis a expressões de violência em nível mundial, devido a posição desigual de poder perante o adulto $^{1}$. A proteção dessa população é uma demanda reconhecida pela Convenção sobre os Direitos da Criança da Organização das Nações Unidas (ONU), que prioriza os direitos humanos básicos da criança, dada sua situação de dependência. Os países signatários da ONU têm o compromisso de elaborar leis e políticas para garantir os direitos do grupo, além de implementar padrões mínimos de suporte ${ }^{2}$.

No Brasil, os direitos de crianças e adolescentes foram garantidos, por meio do Estatuto da Criança e do Adolescente (ECA), que tornou obrigatória a notificação pelos profissionais e responsáveis da área da saúde e da educação de casos suspeitos ou confirmados de maus-tratos (art. 13), prevendo pena para a não comunicação dos casos aos órgãos de proteção (art. 245) ${ }^{3}$. Este procedimento também é assegurado, no âmbito do setor saúde, pela Portaria no 1.968/2001, que institucionalizou a notificação compulsória de maus-tratos contra crianças e adolescentes, atendidos no Sistema Único de Saúde (SUS ${ }^{4}$ e pela Portaria no 104/2011 que dispõe sobre a violência doméstica, sexual e/ou outras violências como o $45^{\circ}$ evento de notificação compulsória ${ }^{5}$.

A notificação é amplamente reconhecida como uma estratégia fundamental para a garantia dos direitos de crianças e jovens ${ }^{6-8}$. Esse procedimento visa, primordialmente, acionar a rede de proteção social, a fim de cessar situações violentas no plano familiar ou por parte de qualquer agressor. Também representa uma oportunidade de intervenção precoce, minimizando o risco de revitimização e as consequências negativas dos atos. Para o sistema de saúde, a notificação permite o dimensionamento epidemiológico do problema, fornecendo subsídios para a formulação de políticas públicas e para o direcionamento de investimentos em núcleos de vigilância e de assistência ${ }^{9}$.

Nesse contexto, percebe-se que o ato de notificar se constitui no primeiro passo para o enfrentamento da violência que acomete a população infanto-juvenil, possibilitando a interrupção do abuso e desencadeamento de medidas de proteção e assistência a vítimas e familiares, assim como também proporciona informações para avaliação da situação local, favorecendo o entendimento do fenômeno ${ }^{7}$.

$\mathrm{Na}$ rede básica de saúde, a Estratégia Saúde da Família (ESF), por meio da atuação da equi- pe multiprofissional, configura-se em um lugar privilegiado para a prevenção, identificação, notificação e vigilância da violência que acomete crianças e jovens pela proximidade com as famílias em seu contexto territorial ${ }^{10,11}$. Contudo, apesar de todos os avanços nesse campo, a identificação e a notificação de maus-tratos em crianças e adolescentes ainda é um desafio para muitos profissionais e gestores dos serviços de saúde ${ }^{11,12}$.

Cabe ressaltar que, a prática da notificação ainda está desigualmente implantada no Brasil. Pouco se sabe sobre o contexto político e institucional e os padrões adotados para a sua efetiva operacionalização $0^{9,13}$. Nesse sentido, este artigo objetiva como diferencial, no acréscimo do conhecimento sobre o tema, demonstrar os fatores associados à notificação de maus-tratos pelos profissionais do setor saúde no contexto da ESF. Supõem-se que estes resultados possam dar subsídio a reorientação da atenção e gestão no cumprimento da legislação em vigor.

Nessa perspectiva, conhecer os mecanismos que aumentam o ato notificatório na atenção básica, no âmbito das diferentes profissões incumbidas de notificar, contribui com o sistema de informação em saúde, bem como com a sociedade, proporcionando o aprimoramento de políticas públicas; e com a sensibilização para a formação dos profissionais de saúde na aquisição de competências que os conduzam diante deste problema de saúde pública.

Contribuindo com o esclarecimento dessas lacunas, este estudo analisa os fatores associados à notificação de maus-tratos em crianças e adolescentes na atenção básica.

\section{Métodos}

Trata-se de estudo transversal, realizado a partir de informações contidas em questionário autoaplicável preenchido por profissionais de saúde (médicos, enfermeiros e cirurgiões-dentistas) acerca da notificação de maus-tratos em crianças e adolescentes. O estudo foi realizado em três Regiões de Saúde do Estado do Ceará (Brejo Santo, Crato e Juazeiro do Norte), no período de julho/2011 a maio/2012. Uma Região de Saúde configura-se em um espaço geográfico contínuo constituído por agrupamentos de municípios limítrofes, com a finalidade de integrar a organização, o planejamento e a execução de ações e serviços de saúde ${ }^{14}$. As Regiões investigadas englobam uma população de 922.929 habitantes, abrangendo 28 municípios. 
de Saúde. Entre 757 profissionais, um tota de 381 respondeu à pesquisa (50,3\%). Desses, 207 enfermeiros (54,3\%), 91 médicos $(23,9 \%)$ e 83 cirurgiões-dentistas $(21,8 \%)$.

Para minimizar as perdas do processo de seleção da amostra, que seria inicialmente censitária, calculou-se a amostra necessária para representar a população-alvo, levando-se em consideração eventos de prevalência aproximada de $16,9 \%{ }^{13}$, suficiente para detectar uma variação de $5 \%$ com nível de confiança em 95\%. Ao considerar eventuais perdas de $10 \%$, o tamanho mínimo da amostra foi calculado em 371 profissionais de saúde, o que supre a quantidade de respostas obtidas no estudo.

O questionário autoaplicável foi composto por 32 questões fechadas, adaptadas e revisadas em trabalho anterior ${ }^{12}$ sobre dados sociodemográficos, formação profissional, instrumentação e conhecimento sobre o tema, identificação e notificação de situações de maus-tratos em crianças e adolescentes.

A operacionalização da coleta de dados obedeceu ao seguinte fluxo: (i) contato com as Coordenadorias Regionais de Saúde (CRES) em foco e encaminhamento da carta de apresentação da pesquisa, contendo a relevância e os objetivos do estudo; (ii) estabelecimento de parcerias com os coordenadores para o desenvolvimento da coleta de dados; (iii) participação de um dos autores em reunião da Comissão Intergestora Bipartite (CIB) das Regiões de Saúde, onde foi descrito para os gestores em saúde municipais os objetivos do estudo e pactuada a entrega dos instrumentos aos profissionais; (iv) os questionários, as cartas de informação ao participante e os termos de consentimento livre e esclarecido, previamente organizados em envelopes lacrados e identificados por municípios, atendendo o quantitativo de participantes, foram entregues aos gestores municipais; também se firmou o compromisso de devolução dos questionários para as respectivas CRES no prazo de 30 dias; (v) retorno dos instrumentos coletados seguindo o fluxo inverso até a chegada a cada Região de Saúde e recolhi- mento do material. Vale destacar que o prazo estipulado não foi atendido pela maioria dos municípios, levando uma das autoras a acompanhar a devolução dos questionários semanalmente e a realizar contatos telefônicos com os gestores em saúde dos municípios.

Estabeleceu-se como desfecho a notificação de maus-tratos em crianças e adolescentes; como variáveis preditoras: profissão (enfermeiro/médico-dentista); sexo (masculino/feminino); idade (22 a 35 anos/> 35 anos); estado civil (casado/ não casado); tempo de formado (até 10 anos/ acima de 10 anos); pós-graduação (área da saúde pública-coletiva/áreas específicas); tempo de trabalho na ESF (até 10 anos/acima de 10 anos) e, ainda, as seguintes variáveis estudadas com respostas dicotômicas (sim/não): participou de treinamento; conhece o ECA; conhece a ficha de notificação; unidade de saúde possui a ficha de notificação; confia nos órgãos de proteção; sabe para onde encaminhar os casos; medo de envolvimento legal; lê sobre a temática; assunto é discutido no trabalho; conhece instituição de assistência à vítima; vantagem instituir a ficha.

Consideraram-se tais variáveis preditoras relevantes à notificação pelos profissionais de saúde de maus-tratos em crianças e adolescentes. Acredita-se que a notificação pode ser influenciada pelo acesso a informação, maior conhecimento sobre o tema, características sociodemográficas, tempo de formado e de trabalho na ESF.

Para analisar a associação entre o desfecho e as variáveis preditoras, empregou-se o teste Exato de Fisher, ao nível de significância de $95 \%$. Seguiram-se a estas análises os procedimentos de modelagem múltipla por meio de regressão logística, incluindo no modelo todas as variáveis preditoras que mostraram associação ao desfecho com significância $\mathrm{p}<0,25$. Permaneceram no modelo múltiplo as variáveis ao nível de significância $\mathrm{p}<$ 0,05 . A força de associação entre as variáveis preditoras e desfecho foi expressa em valores estimados de Odds Ratios (OR) brutos e ajustados, com intervalo de confiança de $95 \%$, após o controle dos fatores potencialmente confundidores. Os dados foram digitados no programa SPSS (SPSS Inc., Chicago, Estados Unidos), versão 16.0. Foram conferidos todos os questionários inseridos, assim como, a consistências dos dados. Para análise, utilizou-se o programa STATA (Stata Corp LP, College Station, TX 77845, USA), versão 11.0.

A pesquisa foi aprovada pelo Comitê de Ética em Pesquisa da Universidade de Fortaleza - UNIFOR. 


\section{Resultados}

A média de idade dos profissionais foi de 35,1 anos (DP $\pm 9,8)$. Observou-se o predomínio do sexo feminino $(64,0 \%)$, da faixa etária de 22 a 35 anos (64,7\%) e de casados (56,5\%). A maior parte tinha até 10 anos de formado $(68,2 \%)$ e possuía tempo de trabalho na ESF de até 10 anos $(80,8 \%)$. Dentre os respondentes, $20,2 \%$ não tinham pós-graduação e $79,8 \%$ possuíam. Dos que referiram ter alguma pós-graduação, predominou os profissionais $(63,1 \%)$ com especialização na área de saúde pública ou coletiva.

Quanto a identificação e notificação de casos de maus-tratos em crianças e adolescentes, $59,3 \%$ afirmaram que não haviam identificado situações de maus-tratos na sua prática profissional. Dos $40,7 \%$ que já tinham identificado algum caso, $65,2 \%$ não notificaram as ocorrências e $34,8 \%$ realizaram esse procedimento. A frequência da notificação foi de 17,0\%.

As variáveis preditoras (profissão, sexo, idade, estado civil, tempo de formado, pós-graduação e tempo de trabalho na ESF) não apresentaram associação com o desfecho $(\mathrm{p}>0,05)$ (Tabela 1$)$.
Participar de treinamento sobre a temática, conhecer a ficha de notificação, unidade de saúde ter a ficha, saber para onde encaminhar os casos e ler sobre a temática apresentaram associação estatisticamente significante com a notificação de maus-tratos $(p<0,05)$, enquanto as demais variáveis não apresentaram diferença estatística (Tabela 2).

As variáveis selecionadas para a análise múltipla foram: participou de treinamento, conhece a ficha de notificação, ficha de notificação na UBS, sabe para onde encaminhar os casos, lê sobre a temática, assunto é discutido no trabalho, conhece instituição que assiste à vítima, acha vantagem instituir a ficha de notificação na atenção básica.

Permaneceram associados positivamente ao desfecho: participar de treinamento, conhecer a ficha de notificação e saber para onde encaminhar os casos. O modelo logístico final mostrou que há mais chance de o profissional notificar casos de maus-tratos em crianças e adolescentes se tiver participado de treinamento específico na área $(2,18$ vezes mais que aqueles que não participaram); se conhecer a ficha de notificação (3,03 vezes mais que a chance daqueles que não conhe-

Tabela 1. Análise bivariada entre notificação de maus-tratos, dados sociodemográficos e formação profissional. Macrorregião de Saúde de Cariri, Ceará, 2012.

\begin{tabular}{|c|c|c|c|c|c|c|}
\hline \multirow{3}{*}{ Variável } & \multicolumn{4}{|c|}{ Notificou maus-tratos } & \multirow{3}{*}{$\begin{array}{c}\text { Não ajustada } \\
\text { OR (IC95\%) }\end{array}$} & \multirow[b]{3}{*}{$\mathbf{p}$} \\
\hline & \multicolumn{2}{|c|}{ Sim } & \multicolumn{2}{|c|}{ Não } & & \\
\hline & $\mathbf{n}$ & $\%$ & $\mathbf{n}$ & $\%$ & & \\
\hline \multicolumn{7}{|l|}{ Profissão $(\mathrm{n}=187)$} \\
\hline Enfermeiro & 39 & 60,0 & 62 & 50,8 & $0,68(0,35-1,32)$ & 0,230 \\
\hline Médico/Dentista & 26 & 40,0 & 60 & 49,1 & & \\
\hline \multicolumn{7}{|l|}{ Sexo $(n=187)$} \\
\hline Masculino & 19 & 29,2 & 49 & 40,2 & & \\
\hline Feminino & 46 & 70,8 & 73 & 59,8 & $0,61(0,30-1,22)$ & 0,138 \\
\hline \multicolumn{7}{|l|}{ Idade $(\mathrm{em}$ anos $)(\mathrm{n}=166)$} \\
\hline $22-35$ & 30 & 53,6 & 65 & 59,1 & & \\
\hline$>35$ & 26 & 46,4 & 45 & 40,9 & $0,79(0,39-1,61)$ & 0,496 \\
\hline \multicolumn{7}{|l|}{ Estado civil $(\mathrm{n}=186)$} \\
\hline Casado & 40 & 61,5 & 70 & 57,8 & $1,16(0,60-2,27)$ & 0,625 \\
\hline Não casado & 25 & 38,5 & 51 & 42,2 & & \\
\hline \multicolumn{7}{|l|}{ Tempo de formado $(\mathrm{n}=187)$} \\
\hline Até 10 anos & 33 & 50,8 & 78 & 63,9 & & \\
\hline Acima de 10 anos & 32 & 49,2 & 44 & 36,1 & $0,58(0,30-1,12)$ & 0,080 \\
\hline \multicolumn{7}{|l|}{ Pós-Graduação* (n = 154) } \\
\hline Área Saúde Pública/Coletiva & 34 & 60,7 & 67 & 68,4 & $0,71(0,34-1,50)$ & 0,336 \\
\hline Áreas específicas & 22 & 39,3 & 31 & 31,6 & & \\
\hline \multicolumn{7}{|c|}{ Tempo de trabalho na $\operatorname{ESF}^{* *}(\mathrm{n}=187)$} \\
\hline Até 10 anos & 45 & 69,2 & 93 & 76,2 & & \\
\hline Acima de 10 anos & 20 & 30,8 & 29 & 23,8 & $0,70(0,34-1,46)$ & 0,300 \\
\hline
\end{tabular}

"Profissionais que referiram possuir pós-graduação. "ESF - Estratégia Saúde da Família. 
Tabela 2. Análise bivariada entre notificação de maus-tratos e fatores associados. Macrorregião de Saúde de Cariri, Ceará, 2012.

\begin{tabular}{|c|c|c|c|c|c|c|}
\hline \multirow{3}{*}{ Variável } & \multicolumn{4}{|c|}{ Notificou maus-tratos } & \multirow{3}{*}{$\begin{array}{c}\text { Não ajustada } \\
\text { OR (IC95\%) }\end{array}$} & \multirow[b]{3}{*}{$\mathbf{p}$} \\
\hline & \multicolumn{2}{|c|}{ Sim } & \multicolumn{2}{|c|}{ Não } & & \\
\hline & $\mathbf{n}$ & $\%$ & n & $\%$ & & \\
\hline \multicolumn{7}{|c|}{ Participou de treinamento $(\mathrm{n}=185)$} \\
\hline Sim & 34 & 52,3 & 28 & 23,3 & \multirow[t]{2}{*}{$3,60(1,79-7,22)$} & \multirow[t]{2}{*}{$<0,001$} \\
\hline Não & 31 & 47,7 & 92 & 76,7 & & \\
\hline \multicolumn{7}{|l|}{ Conhece o $\mathrm{ECA}^{*}(\mathrm{n}=187)$} \\
\hline $\operatorname{Sim}$ & 54 & 83,1 & 93 & 76,2 & \multirow{2}{*}{$1,53(0,67-3,67)$} & \multirow{2}{*}{0,276} \\
\hline Não & 11 & 16,9 & 29 & 23,8 & & \\
\hline \multicolumn{7}{|c|}{ Conhece a ficha de notificação $(\mathrm{n}=185)$} \\
\hline Sim & 44 & 68,8 & 41 & 33,9 & \multirow[t]{2}{*}{$4,29(2,14-8,69)$} & \multirow[t]{2}{*}{$<0,001$} \\
\hline Não & 20 & 31,2 & 80 & 66,1 & & \\
\hline \multicolumn{7}{|c|}{ Ficha de notificação na $\operatorname{UBS}^{* *}(\mathrm{n}=181)$} \\
\hline Sim & 25 & 40,3 & 21 & 17,6 & \multirow[t]{2}{*}{$3,15(1,48-6,68)$} & \multirow[t]{2}{*}{$<0,001$} \\
\hline Não & 37 & 59,7 & 98 & 82,4 & & \\
\hline \multicolumn{7}{|c|}{ Confia nos órgãos de proteção $(\mathrm{n}=180)$} \\
\hline $\operatorname{Sim}$ & 43 & 69,3 & 74 & 62,7 & \multirow{2}{*}{$1,34(0,66-2,76)$} & \multirow{2}{*}{0,374} \\
\hline Não & 19 & 30,7 & 44 & 37,3 & & \\
\hline \multicolumn{7}{|c|}{ Sabe para onde encaminhar $(\mathrm{n}=185)$} \\
\hline Sim & 57 & 89,1 & 83 & 68,6 & \multirow[t]{2}{*}{$3,72(1,49-10,53)$} & \multirow[t]{2}{*}{0,002} \\
\hline Não & 07 & 10,9 & 38 & 31,4 & & \\
\hline \multicolumn{7}{|c|}{ Tem medo de envolvimento legal $(n=179)$} \\
\hline Sim & 24 & 38,7 & 52 & 44,4 & & \\
\hline Não & 38 & 61,3 & 65 & 55,6 & $0,78(0,39-1,54)$ & 0,460 \\
\hline \multicolumn{7}{|l|}{ Lê sobre a temática $(\mathrm{n}=186)$} \\
\hline Sim & 34 & 52,3 & 42 & 34,7 & \multirow[t]{2}{*}{$2,06(1,06-3,99)$} & \multirow[t]{2}{*}{0,019} \\
\hline Não & 31 & 47,7 & 79 & 65,3 & & \\
\hline Assunto é discutido no trabal & & & & & & \\
\hline $\operatorname{Sim}$ & 38 & 58,5 & 54 & 44,3 & $1,77(0,92-3,41)$ & 0,064 \\
\hline Não & 27 & 41,5 & 68 & 55,7 & & \\
\hline Conhece instituição assiste à & & & & & & \\
\hline $\operatorname{Sim}$ & 21 & 33,3 & 26 & 21,5 & $1,82(0,86-3,80)$ & 0,080 \\
\hline Não & 42 & 66,7 & 95 & 78,5 & & \\
\hline Acha vantagem instituir a fich & & & & & & \\
\hline Sim & 60 & 92,3 & 103 & 85,1 & $2,09(0,70-7,57)$ & 0,155 \\
\hline Não & 05 & 7,7 & 18 & 14,9 & & \\
\hline
\end{tabular}

*ECA - Estatuto da Criança e do Adolescente. "* UBS - Unidade Básica de Saúde.

cem a ficha) e se o profissional souber para onde encaminhar os casos $(2,55$ vezes mais que os que não sabem para onde encaminhar) (Tabela 3 ).

Confirmou-se a variável participar de treinamento como potencial fator de confundimento na relação entre notificar maus-tratos e conhecer a ficha de notificação.

\section{Discussão}

Os resultados do estudo evidenciaram o distanciamento entre identificar maus-tratos em crian- ças e adolescentes e proceder com a notificação dos casos. A frequência da notificação encontrada $(17,0 \%)$ é baixa, tendo em vista a obrigatoriedade desse ato preconizado pelo ECA e pela Portaria no 104/2011 do Ministério da Saúde ${ }^{3,5}$.

Estudos realizados em outros países e no Brasil não se mostraram diferentes. No Canadá, apenas $10,0 \%$ das comunicações enviadas para os serviços de proteção foram relatadas por trabalhadores da saúde ${ }^{15}$. Nos EUA, pesquisa envolvendo as unidades federativas constatou que 66,6\% dos casos suspeitos de maus-tratos não foram comunicados aos órgãos de proteção pelos 
Tabela 3. Análise multivariada entre notificação de maus-tratos e fatores associados. Macrorregião de Saúde de Cariri, Ceará, 2012.

\begin{tabular}{|c|c|c|c|c|c|c|}
\hline \multirow{3}{*}{ Variável } & \multicolumn{4}{|c|}{ Notificou maus-tratos } & \multirow{3}{*}{$\begin{array}{c}\text { Não ajustada } \\
\text { OR (IC95\%) }\end{array}$} & \multirow[b]{3}{*}{$\mathbf{p}$} \\
\hline & \multicolumn{2}{|c|}{ Sim } & \multicolumn{2}{|c|}{ Não } & & \\
\hline & $\mathbf{n}$ & $\%$ & n & $\%$ & & \\
\hline \multicolumn{7}{|c|}{ Participou de treinamento $(\mathrm{n}=185)$} \\
\hline $\operatorname{Sim}$ & 34 & 52,3 & 28 & 23,3 & $2,18(1,05-4,53)$ & 0,035 \\
\hline Não & 31 & 47,7 & 92 & 76,7 & & \\
\hline \multicolumn{7}{|c|}{ Conhece a ficha de notificação $(\mathrm{n}=185)$} \\
\hline Sim & 44 & 68,8 & 41 & 33,9 & $3,03(1,47-6,24)$ & 0,003 \\
\hline Não & 20 & 31,2 & 80 & 66,1 & & \\
\hline \multicolumn{7}{|c|}{ Sabe para onde encaminhar $(\mathrm{n}=185)$} \\
\hline Sim & 57 & 89,1 & 83 & 68,6 & $2,55(1,01-6,43)$ & 0,047 \\
\hline Não & 07 & 10,9 & 38 & 31,4 & & \\
\hline
\end{tabular}

" Profissionais que referiram possuir pós-graduação. “ESF - Estratégia Saúde da Família.

profissionais com obrigação legal de notificar ${ }^{16}$. Na Jordânia, investigação com 400 dentistas, verificou que 50,0\% suspeitaram de alguma forma de abuso, no entanto apenas 12,0\% notificaram suas suspeitas ${ }^{17}$. No cenário brasileiro, Assis et al. ${ }^{13}$ destacam o baixo percentual nacional $(16,9 \%)$ de notificações, baseado nos registros de violência doméstica, sexual e outras violências contra crianças (0-9 anos) do Sistema de Informação de Agravos de Notificação (Sinan).

Esses dados sugerem que o ato de notificar maus-tratos não se configura como uma prática internalizada na rotina dos profissionais do setor saúde, mesmo em países onde a notificação foi estabelecida há aproximadamente 40 anos e os sistemas de atendimento são mais aprimorados. Percebe-se que a notificação circunscreve aspectos que extrapolam questões relativas à estrutura e aos dispositivos legais que embasam essa prática. Em vista disto, algumas indagações emergem: quais as dificuldades inerentes à operacionalização do ato de notificar maus-tratos em crianças e adolescentes? Quais as insuficiências do sistema de saúde para a efetivação desse procedimento?

Alguns encaminhamentos a estas questões são que fatores culturais, medo de envolvimento legal, ausência de proteção, fragilidades na formação, insuficiência de processos de avaliação, supervisão e apoio profissional podem influenciar a decisão do profissional, notificar ou não um caso.

Culturalmente, o uso da violência, como forma de educação dos filhos, ainda é aceito na sociedade, fazendo com que práticas abusivas, muitas vezes, sejam consideradas como naturais pelos profissionais de saúde. Além disso, há o receio de adentrar na intimidade da família e ser testemunha de fatos que, por ventura, possam ter desdobramentos legais.

No contexto da atenção básica, outra relevante dificuldade para a operacionalização da notificação consiste no medo de envolvimento legal. Os profissionais que trabalham na ESF, pela proximidade territorial com a comunidade, estão mais expostos à retaliação por parte do autor da agressão. Oliveira et al. ${ }^{18}$ chamam a atenção para as equipes de saúde da família que, muitas vezes, atuam em comunidades consideradas violentas, onde são submetidas a "regras de convivência" estabelecidas pelas pessoas que dominam o local através do terror e do medo. Atrelado a isso, a situação ainda é agravada pela ausência de proteção e suporte institucional para essa iniciativa. Dessa forma, os profissionais acabam se omitindo do processo em nome da convivência profissional e, da própria sobrevivência.

Outro ponto configura-se na precária formação dos recursos humanos em saúde sobre a violência que acomete os grupos vulneráveis, incluindo crianças e adolescentes. Esse assunto não é abordado na maioria das matrizes curriculares dos cursos de graduação e pós-graduação da saúde, e não se apresenta como alvo de capacitação na formação permanente das equipes de saúde da família. Por esse motivo, muitos profissionais apresentam fragilidades na condução dos casos, podendo levar à revitimização da criança ou do jovem e ao aumento dos danos causados pela violência. 
As ações da gestão na atenção básica, em relação à notificação de maus-tratos, devem extrapolar a mera distribuição das fichas às unidades de saúde. O modo como ocorre o suporte para a equipe de saúde na identificação, no manejo e acompanhamento das situações de violência ainda não é adequado, necessitando mais investimentos da gestão em processos de formação, avaliação, supervisão e apoio ao profissional incumbido de notificar. Também é oportuno evidenciar que, apesar de a não comunicação de casos suspeitos ou confirmados de maus-tratos em crianças e adolescentes ser passível de pena (multa de três a 20 salários de referência, aplicando-se o dobro em caso de reincidência) ${ }^{3}$, a aplicação dessa penalidade não é verificada nos contextos da produção de cuidado em saúde.

Estes fatos faz-nos reportar que muitas vezes, no Brasil, a operacionalização de políticas "esbarra" na escassez de recursos, na carência de processos de capacitação, acompanhamento, supervisão e avaliação das ações, comprometendo uma postura eficaz por parte dos profissionais da “ponta” diante de situações de violência. Embora tenhamos avançado muito quanto ao arcabouço legal, à concretização do ato notificatório de maus-tratos em criança e adolescentes se mostra complexo, necessitando de grandes debates na sociedade.

O presente estudo elucida os fatores que facilitam a efetivação desse ato pelos recursos humanos em saúde na realidade estudada. A análise múltipla mostrou que participar de treinamento sobre o tema, conhecer a ficha de notificação e saber para onde encaminhar os casos aumentou a chance de os profissionais notificarem situações de violência contra crianças e adolescente. $\mathrm{Na}$ análise de potenciais variáveis de confundimento, participar de treinamento foi determinante para o profissional conhecer a ficha e, consequentemente, notificar os casos.

Percebe-se que os fatores que elevam a notificação de maus-tratos estão relacionados à formação e capacitação dos trabalhadores sobre o assunto, favorecendo a apropriação de conhecimentos que os instrumentalizem para efetivar o ato notificatório. Nesta perspectiva, lidar com questões referentes à violência contra crianças e adolescentes demanda a construção de novas compreensões no âmbito da atenção básica visando à qualificação dos processos de trabalho e das práticas profissionais.

Esses resultados correlacionam-se com o que tem sido relatado por outros autores nos Estados Unidos, Austrália, Irlanda, Jordânia e Brasil, reforçando o pressuposto da influência positiva do conhecimento, do acesso à capacitação e da qualificação da formação sobre a atitude dos profissionais de notificar ${ }^{12,17,19-22}$.

Investigação nos EUA concluiu que médicos da atenção primária que receberam educação formal sobre maus-tratos à criança eram 10 vezes mais propensos a relatar casos suspeitos ou confirmados de abuso aos órgãos de proteção do que os que não tiveram treinamento formal ${ }^{20}$. Sonbol et al. ${ }^{17}$ também verificaram, na Jordânia, que a educação formal foi um fator preditivo para a notificação de maus-tratos por dentistas (os profissionais capacitados tinham três vezes mais probabilidade de relatar o caso do que aqueles sem treinamento). Em um estudo transversal empreendido com 930 enfermeiros australianos, o treinamento esteve relacionado à maior probabilidade de intenções de notificar abuso infantil e negligência ${ }^{21}$. Leite et al. ${ }^{22}$ pesquisando e intervindo com médicos residentes no Estado de São Paulo, analisaram o número de notificações de maus-tratos infantis antes e após a realização de um curso sobre o tema. Foi constatado que houve um aumento considerável da quantidade de notificações após a realização do treinamento, mas ocorreu uma diminuição nos meses seguintes, indicando a necessidade de educação continuada.

Esses dados ratificam a necessidade de educação permanente com enfoque na violência contra crianças e adolescentes. Contudo, para se evitar uma prática puramente técnica, essa intervenção não deve se limitar apenas aos aspectos fisiopatológicos, sendo primordial também abordar aspectos tais como: fatores de risco e de proteção; reconhecimento dos sinais de violência; parâmetros legais; procedimento de notificação e encaminhamentos; papel dos órgãos e da rede de proteção; e acompanhamento das vítimas.

Uma vez que a violência contra os grupos vulneráveis se configura em uma demanda cada vez maior na rotina dos serviços de saúde e existe a obrigatoriedade legal de notificar essas situações pelos trabalhadores da saúde, esta intervenção educacional deveria ser introduzida na formação acadêmica, desde o período da graduação, sendo oportuno contemplar conteúdos relacionados com a temática nas matrizes curriculares dos cursos da área da saúde. Alvarez et al. ${ }^{23}$ advogam que as informações sobre os maus-tratos devem ser incorporadas nos programas de formação em vários níveis, incluindo os cursos de graduação, pós-graduação e a educação permanente proporcionada pelos serviços, com objetivo de efetivar o cumprimento da legislação vigente e maximizar 
o compromisso com a notificação, garantindo os direitos de crianças e adolescentes.

No nível da educação permanente no contexto da atenção básica, o investimento na formação profissional é uma medida imperativa, tendo o potencial para transformar práticas e modificar o cenário de subnotificações. Em contrapartida, evidencia-se que a lacuna do conhecimento ultrapassa os limites das instituições formadoras e se estende para a rotina das unidades de saúde, em virtude da temática da violência ser ainda pouco trabalhada, ou até mesmo, estar ausente da pauta de educação permanente dos serviços.

Como resultado se vê, em muitas situações, o grande distanciamento entre o que é oferecido pelos serviços e o que está posto nas Políticas Nacionais de Saúde. Enfrentar os conflitos e contradições presentes hoje no SUS depende do protagonismo dos múltiplos atores que o compõe. Os trabalhadores de saúde, que poderiam desempenhar um papel fundamental nessa luta, muitas vezes não são suficientemente formados e preparados, e isso se torna um fator que fragiliza diversos aspectos da assistência, da organização e gestão das redes de serviços ${ }^{24}$.

Na concepção de Mendonça et al..$^{25}$, o desenvolvimento contínuo dos profissionais das equipes de saúde da família e as estratégias de educação permanente facilitam a qualificação dos trabalhadores para o exercício de suas funções e para o uso de diretrizes assistenciais, além de contribuir com a melhora da resolutividade da Atenção Primária à Saúde.

A consolidação da Política Nacional de Educação Permanente em Saúde (PNEPS) funciona como dispositivo transformador desse contexto, na medida em que favorece mudanças nas práticas do trabalho em saúde e articula o sistema de saúde com as instituições formadoras na identificação de problemas cotidianos, para a formulação de processos educativos que respondam a realidade e as necessidades do SUS ${ }^{26}$.

Segundo a PNEPS, a educação permanente se baseia na aprendizagem significativa e na possibilidade de transformar as práticas profissionais. Também propõe que os processos de educação dos trabalhadores da saúde se desenvolvam a partir da problematização do processo de trabalho, e considera que as necessidades de formação e desenvolvimento dos profissionais sejam embasadas pelas necessidades de saúde das pessoas e populações, tendo como objetivos a transformação das práticas profissionais e da própria organização do trabalho ${ }^{26}$.
Diante dos altos índices de violência nos territórios adscritos, a ESF, que assume em um dos seus pilares a responsabilidade pelas demandas da comunidade, deve trabalhar a demanda da violência como uma questão prioritária. O fenômeno pede atenção integral e diferenciada com exaustivos investimentos nas melhorias dos determinantes sociais que alimentam o ciclo da violência. Trilhar nesta ótica é consolidar práticas já instituídas, como o ato de notificar.

Dessa maneira, a capacitação dos recursos humanos em saúde se torna uma conduta estratégica para produzir mudanças nas suas práticas, visando o investimento na promoção de ações que os sensibilize quanto aos direitos e às necessidades das crianças e adolescentes, propiciando um aprimoramento profissional para identificar casos de maus-tratos, e ampliar o compromisso da notificação.

Também é importante salientar, que os programas de educação permanentes necessitam ser avaliados sistematicamente, averiguando o alcance dessa formação, a metodologia empregada, a forma de explanação e envolvimento dos que estão sendo capacitados, bem como o monitoramento de transformação de realidades. Corrobora a assertiva a afirmação de que formar a equipe de saúde da família, como um todo, é mais eficiente do que treinar os trabalhadores individualmente, a fim de reduzir a disparidade de conhecimento dos profissionais que trabalham na mesma instituição ${ }^{27}$.

Além da capacitação formal, outras estratégias também poderiam ser utilizadas para auxiliar os profissionais de saúde na prática notificatória. Países como os EUA, Canadá e Austrália utilizam sites específicos para orientar os trabalhadores da saúde. Esses sites disponibilizam informações sobre legislação, serviços de assistência à vítima e órgãos de proteção ${ }^{28}$. Outro recurso como o treinamento online, adaptado para o setor saúde, tem mostrado resultados promissores no Reino Unido $^{29}$. O uso de tais métodos de suporte e treinamento poderia ser um dos diferenciais na realidade brasileira.

Assume-se como limitação o modo como se desenvolveu a coleta de dados, por meio do envio de questionários para os profissionais que compõem a equipe básica da ESF, com monitoramento na devolução dos mesmos por uma das autoras. Dessa forma, os profissionais que participaram do estudo podem não representar, em sua totalidade, a prática dos profissionais das Regiões de Saúde estudadas; infere-se que essa participa- 
ção possa ter sido influenciada por: (i) interesse pessoal pelo tema; (ii) maior comprometimento com as ações de saúde na atenção básica; (iii) conhecimento/capacitação prévios. Por outro lado, pode-se supor que os profissionais que optaram por não participar da pesquisa não o fizeram por desconhecer a legislação que ancora o processo de trabalho na atenção básica. Mesmo com esses possíveis vieses, os achados apontaram fragilidades no ato de notificar.

O estudo conclui que participar de treinamento sobre o tema, conhecer a ficha de notificação e saber encaminhar os casos são mecanismos que aumentam a notificação de maus-tratos em

\section{Colaboradores}

GAR Moreira e LJES Vieira participaram de todas as etapas da elaboração do artigo. IS Gama trabalhou na metodologia, análise estatística e interpretação dos dados. SF Deslandes, MAJ Pordeus e AVM Brilhante contribuíram com a revisão crítica do artigo.

\section{Agradecimentos}

À Fundação Cearense de Apoio ao Desenvolvimento Científico e Tecnológico (FUNCAP), ao Conselho Nacional de Desenvolvimento Científico e Tecnológico (CNPq), Bolsa Produtividade em Pesquisa; e à Coordenação de Aperfeiçoamento de Pessoal de Nível Superior (CAPES), Bolsa Mestrado. crianças e adolescentes pelos profissionais que atuam na atenção básica; o que sinaliza associação direta do ato de notificar com questões relativas ao conhecimento e capacitação na temática.

Esses dados fornecem subsídios para o planejamento de estratégias que visem à efetiva operacionalização da notificação de maus-tratos em crianças e adolescentes. Dessa forma, sugere-se instituições de ensino e, à gestão do setor saúde, o incremento do tema na formação dos profissionais de saúde e que programas de educação permanente sejam desenvolvidos e sistematicamente avaliados com a finalidade de favorecer a qualificação da prática profissional.

\section{Referências}

1. Krug EG, Dahlberg LL, Mercy JA, Zwi AB, Lozano R, editores. Relatório mundial sobre violência e saúde. Geneva: Organização Mundial da Saúde (OMS); 2002.

2. Fundo das Nações Unidas para a Infância (UNICEF). A Convenção sobre os direitos da Criança. Brasília: UNICEF; 1989.

3. Brasil. Lei 8.069, de 13 de julho de 1990. Dispõe sobre o Estatuto da Criança e do Adolescente e dá outras providências. Diário Oficial da União 1990; 16 jul.

4. Brasil. Ministério da Saúde. Portaria GM/MS no 1.968, de 25 de outubro de 2001. Dispõe sobre a notificação, às autoridades competentes, de casos de suspeita ou de confirmação de maus-tratos contra crianças e adolescentes atendidos nas entidades do Sistema Único de Saúde. Diário Oficial da União 2001; 25 out.

5. Brasil. Ministério da Saúde (MS). Portaria nº 104, de 25 de janeiro de 2011. Define as terminologias adotadas em legislação nacional, a relação de doenças, agravos e eventos em saúde pública de notificação compulsória em todo território nacional e estabelece fluxos, critérios, responsabilidades e atribuições aos profissionais de saúde. Diário Oficial da União 2011; 25 jan.

6. Coohey C, Renner LM, Hua L, Zhang YJ, Whitney SD. Academic achievement despite child maltreatment: A longitudinal study. Child Abuse Negl 2011; 35(9):688699.

7. Deslandes S, Mendes CHF, Lima JS, Campos DS. Indicadores das ações municipais para a notificação e o registro de casos de violência intrafamiliar e exploração sexual de crianças e adolescentes. Cad Saude Publica 2011; 27(8):1633-1645. 
8. Yampolskava S, Armstrong MI, King-Miller T. Contextual and individual-level predictors of abused children's reentry into out-of-home care: A multilevel mixture survival analysis. Child Abuse Negl 201 1; 35(9):670-679.

9. Lima JS, Deslandes SF. A notificação compulsória do abuso sexual contra crianças e adolescentes: uma comparação entre os dispositivos americanos e brasileiros. Interface (Botucatu) 2011; 15(38):819-832.

10. Lima MCCS, Costa MCO, Bigras M, Santana MAO, Alves TDB, Nascimento OC, Silva MR. Atuação profissional da atenção de saúde face à identificação e notificação da violência infanto-juvenil. Rev. Baiana de Saúde Pública 2011; 35(Supl. 1):118-137.

11. Lobato GR, Moraes CL, Nascimento MC. Desafios da atenção à violência doméstica contra crianças e adolescentes no Programa Saúde da Família em cidade de médio porte do Estado do Rio de Janeiro, Brasil. Cad Saude Publica 2012; 28(9):1749-1758.

12. Luna GLM, Ferreira RC, Vieira LJES. Notificação de maus-tratos em crianças e adolescentes por profissionais da Equipe Saúde da Família. Cien Saude Colet 2010; 15(2):481-491.

13. Assis SG, Avanci JQ, Pesce RP, Pires TO, Gomes DL. Notificações de violência doméstica, sexual e outras violências contra crianças no Brasil. Cien Saude Colet 2012; 17(9):2305-2317.

14. Brasil. Decreto no 7.508, de 28 de junho de 2011. Regulamenta a Lei no 8.080, de 19 de setembro de 1990, para dispor sobre a organização do Sistema Único de Saúde - SUS, o planejamento da saúde, a assistência à saúde e a articulação interfederativa, e dá outras providências. Diário Oficial da União 2011; 29 jun.

15. Tonmyr L, Li YA, Williams G, Scott D, Jack SM. Patterns of reporting by health care and non-health care professionals to child protection services in Canada. Paediatr Child Health 2010; 15(8):e25-e32.

16. Sedlak AJ, Mettenburg J, Basena M, Petta I, McPherson K, Green A, Li S. Fourth National Incidence Study of Child Abuse and Neglect (NIS-4): Report to congress. Washington: US Department of Health and Human Resources, Administration for Children and Families; 2010.

17. Sonbol HN, Abu-Ghazaleh S, Rajab LD, Baqain ZH, Saman R, Al-Bitar ZB. Knowledge, educational experiences and attitudes towards child abuse amongst Jordanian dentists. Eur J Dent Educ 2012; 16(1):e158-e165.

18. Oliveira MT, Samico I, Ishigami ABM, Nascimento RMM. Violência intrafamiliar: a experiência dos profissionais de saúde nas Unidades de Saúde da Família de São Joaquim do Monte, Pernambuco. Rev Bras Epidemiol 2012; 15(1):166-178.
19. Lazenbatt A, Freeman R. Recognizing and reporting child physical abuse: a survey of primary healthcare professionals. J Adv Nurs 2006; 56(3):227-236.

20. Flaherty EG, Sege RD, Griffith J, Price LL, Wasserman R, Slora E, Dhepyasuwan N, Harris D, Norton D, Angelilli ML, Abney D, Binns HJ. From suspicion of physical child abuse to reporting: Primary care clinician decision-making. Pediatrics 2008; 122(3):611-619.

21. Fraser JA, Mathews B, Walsh K, Chen L, Dunne M. Factors influencing child abuse and neglect recognition and reporting by nurses: A multivariate analysis. Int J Nurs Stud 2010; 47(2):146-153.

22. Leite CN, Oliveira RKF, Carmerini MB, Ramos C, Moscardini AC. Notificação de maus-tratos infantis: necessidade de educação médica continuada. Arq Ciênc Saúde 2006; 13(1):22-26.

23. Alvarez KM, Kenny MC, Donohue B, Carpin KM. Why are professionals failing to initiate mandated reports of child maltreatment, and are there any empirically based training programs to assist professionals in the reporting process? Aggress Violent Behav 2004; 9(5):563-578.

24. Mângia EF. Política nacional de educação permanente em saúde: desafios e perspectivas [editorial]. Rev. Ter. Ocup. Univ. 2007; 18(3):i-ii.

25. Mendonça MHM, Martins MIC, Giovanella L, Escorel S. Desafios para gestão do trabalho a partir de experiências exitosas de expansão da Estratégia de Saúde da Família. Cien Saude Colet 2010; 15(5):2355-2365.

26. Brasil. Ministério da Saúde (MS). Secretaria de Gestão do Trabalho e da Educação na Saúde. Departamento de Gestão da Educação em Saúde. Política Nacional de Educação Permanente em Saúde. Brasília: MS; 2009.

27. Reece RM, Jenny C. Medical training in child maltreatment. Am J Prev Med 2005; 29(5):266-271.

28. Pietrantonio AM, Wright E, Gibson KN, Alldred T, Jacobson D, Niec A. Mandatory reporting of child abuse and neglect: Crafting a positive process for health professionals and caregivers. Child Abuse Negl 2013; 37(23):102-109.

29. Newton JT, Bower E. The management of abuse: a resource manual for the dental team. London: Stephen Hancocks Limited; 2005.

Artigo apresentado em 14/09/2013

Aprovado em 22/12/2013

Versão final apresentada em 27/12/2013 